International Journal of Pure and Applied Mathematics

Volume $97 \quad$ No. 3 2014, 287-301

ISSN: 1311-8080 (printed version); ISSN: 1314-3395 (on-line version)

url: http://www.ijpam.eu

doi: http://dx.doi.org/10.12732/ijpam.v97i3.3

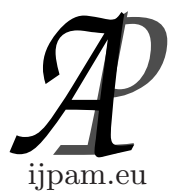

\title{
MELLIN TRANSFORM METHOD FOR SOLVING THE BLACK-SCHOLES EQUATION
}

\author{
F. AlAzemi ${ }^{1} \S$, A. AlAzemi ${ }^{2}$, L. Boyadjiev ${ }^{3}$ \\ ${ }^{1,2,3}$ Department of Mathematics \\ Faculty of Science \\ Kuwait Universiry \\ P.O. Box 5969, Safat, 13060, KUWAIT
}

This paper is dedicated to Prof. Dr. Rudolf Scherer

Karlsruhe Institute of Technology, Germany

on occasion of his $70^{\text {th }}$ birthday.

\begin{abstract}
In this paper, we obtain an analytical solution of the Black-Scholes equation for the European and the American put options by using the Mellin integral transform method. The analytical solution thus obtained of the BlackScholes equation for the European put option is compared with the existing already numerical solutions. By using a join Mellin-Laplace integral transforms method, a solution of a boundary value problem for a fractional Black-Scholes equation is also derived. For some prescribed values of the parameters, computational values of the analytical results obtained are compared with some known numerical solutions.
\end{abstract}

AMS Subject Classification: 62P05, 97M30, 91G60, 44A05

Key Words: Black-Scholes equation, Mellin integral transform, European put option, American put option, option pricing

Received: April 1, 2014

(C) 2014 Academic Publications, Ltd.

${ }^{\S}$ Correspondence author url: www.acadpubl.eu 


\section{Introducation}

Until 1973, the pricing of an option was done by little more than guesswork. Black and Scholes [2], and Merton [14] derived a second order partial differential equation for the value of an option on stocks which then transformed option pricing into a science. Nowadays, The Black-Scholes equation is one of the most important contributions to the field of financial mathematics. The BlackScholes equation is a significant mathematical formula designed to price an option as a function of certain variables.

The European style option and the American style option are the two exercise styles in option trading. The major difference between them is that the American option allows the holder to exercise the option at anytime prior to expiration while the European one allows exercise only upon expiration. In fact, most exchanged options are American options. They are generally more expensive than European options due to the benefit of being able to exercise the options early. This specifically important for companies that are going bunkrupt. Being able to exercise early allows them to sell the shares they posses before these shares get delisted.

A widely used model for pricing of American options is the Black-Scholes model [2], but the actual traded prices are usually higher than theoritical values generated by the model. This is because Black-Scholes options pricing model was designed with European options in mind. Until 2010 there is no generally accepted method of pricing American options even though several methods using regression [20], least square [12], Monte Carlo [7, 19], or binomial [9] have been proposed. A qualitative and quantitative comparison of various analytical and numerical approximation methods for calculating a position of the early exercise boundary of the American put option paying zero dividents is provided in [11].

The idea to employ integral transforms in solving the Black-Scholes equation is not new. For instance Mellin transform was used in $[8,21]$ for deriving a solution to an equation for the price of a European put on a single underlying stock. A method involved Mellin transform was also used in [4] for solving arithmetic Asian options partial differential equations to get closed form solution for a continuous arithmetic Asian option. Laplace homotopy perturbation method is adopted in [10] for optaining a quick and accurate solution to a fractional generalization of the Black-Scholes equation with boundary conditions for a European option pricing problem.

In this paper we are focused on an integral method based on the Mellin integral transform for solving both the Black-Scholes equation for the Eurpean 
and American put options. Although the results obtained are in closed integral form, we confirm their usefulness on some examples by using the computing system Mathematica. We also consider a fractional Black-Scholes equation in terms of the Caputo and Riemann-Liouville fractional derivatives. We obtain its solution in integral form by using a joint Mellin-Laplace integral transforms method.

The presented paper is organized in the following way. In Section 2, we discuss the preliminaries and the formulations of the model. In Section 3, we prove the main theorem of the present paper by using the concept of the Mellin transform for solving the Black-Scholes equation for the European put option and the American put option. We compare our results with those obtained by different numerical techniques in Section 4. The Mellin-Laplace join transforms method for solving a fractional Black-Scholes equation is presented in Section 5 .

\section{Problem Formulation and Some Preliminaries}

Let $S$ denote the price of the stock, $K$ denote the strike of the option (exercise price), $r$ denote the risk-free interest rate, $\sigma$ denote the volatility of the stock's returns, and $t$ denote a time in years (assuming now $t=0$ and expiry $t=T$ ). In such setting, the Black-Scholes equation for the price of an European put option $p_{E}(S, t)$ reads as

$$
\frac{\partial p_{E}}{\partial t}+\frac{\sigma^{2} S^{2}}{2} \frac{\partial^{2} p_{E}}{\partial S^{2}}+r S \frac{\partial p_{E}}{\partial S}-r p_{E}=0,
$$

where $0<t<T$ and $0<S<\infty$ subject to the boundary conditions

$$
\left\{\begin{array}{l}
p_{E}(S, T)=\theta(S)=(K-S)^{+} \\
p_{E}(0, t)=K e^{r(T-t)} \quad \text { (Put-Call Parity) }
\end{array}\right.
$$

and where

$$
\lim _{S \rightarrow \infty} p_{E}(S, t)=0 .
$$

The early exercise feature of American option gives rise to a free boundary value problem. At each instant $t$, the free boundary is a critical stock price $S^{*}(t)$, on one side of which it is optimal to hold the option (this region is called the continuation region) and on the other side it is optimal to exercise it (exercise region). For an American put, the exercise region is given by $0<S<$ $S^{*}$. The option price in this region is equal to the payoff. In the continuation 
region $S>S^{*}$, the option price is the solution of the non-homogeneous BlackScholes equation that for American put option $p_{A}(S, t)$ has the form

$$
\frac{\partial p_{A}}{\partial t}+\frac{\sigma^{2} S^{2}}{2} \frac{\partial^{2} p_{A}}{\partial S^{2}}+r S \frac{\partial p_{A}}{\partial S}-r p_{A}=f(S, t),
$$

where $0<t<T, 0<S<\infty$, and

$$
f(S, t)= \begin{cases}-r K, & \text { if } 0<S<S^{*}, \\ 0, & \text { if } S>S^{*},\end{cases}
$$

subject to the time condition

$$
p_{A}(S, t)=\theta(S)=(K-S)^{+} .
$$

Both, the price of the option $p_{A}(S, t)$ and the free boundary $S^{*}(t)$ are unknown functions to be found by imposing on them the following two conditions. The first one, known as the "Value Matching" condition ensures that the price of the option is continuous across the free boundary. The second condition, known as the "high contact" condition ensures that the slope of this price is continuous on the free boundary. Together these conditions are called "smooth-pasting" conditions and are written respectively as

$$
p_{A}\left(S^{*}, t\right)=\left(K-S^{*}\right)^{+}, \text {and }\left(\frac{\partial p_{A}}{\partial S}\right)_{S=S^{*}}=-1 .
$$

In this paper, we solve equation (1) subject to the boundary conditions stated in equation (2) by using the Mellin integral transform which can be stated in the following form

$$
M\{f(x) ; \eta\}=f^{*}(\eta)=\int_{0}^{\infty} x^{\eta-1} f(x) d x, \quad \gamma_{1}<\operatorname{Re} \eta<\gamma_{2} .
$$

For more detailed information on the Mellin transform, one can refer to special treaties and/or handbooks such as $[5,13,17]$.

Let $M^{-1}\left\{f^{*}(\eta) ; x\right\}$ denote the inverse Mellin integral transform which is defined by

$$
M^{-1}\left\{f^{*}(\eta) ; x\right\}=f(x)=\frac{1}{2 \pi i} \int_{\gamma-i \infty}^{\gamma+i \infty} x^{-\eta} f^{*}(\eta) d \eta,
$$

where $x>0, \gamma=\operatorname{Re} \eta$, and $\gamma_{1}<\gamma<\gamma_{2}$. 
Because of our further considerations, we restrict our attention just to the following basic properties of the Mellin transform,

$$
\begin{gathered}
M\left\{x^{a} f(x) ; \eta\right\}=f^{*}(\eta+a), \\
M\left\{x^{n} f^{(n)}(x) ; \eta\right\}=(-1)^{n} \frac{\Gamma(\eta+n)}{\Gamma(\eta)} f^{*}(\eta),
\end{gathered}
$$

and

$$
M\left\{\int_{0}^{\infty} f(\xi) g\left(\frac{x}{\xi}\right) \frac{d \xi}{\xi} ; \eta\right\}=f^{*}(\eta) g^{*}(\eta) .
$$

These three basic properties are commonly refered to as shifting property (8), transform of differential operator (9), and convolution theorem (10).

The Mellin transform (6) and its inverse (7) can be also derived from the Fourier transform and its inverse, defined respectively by

$$
F\{g(\xi) ; \kappa\}=G(\kappa)=\int_{-\infty}^{\infty} e^{-i \kappa \xi} g(\xi) d \xi,
$$

and

$$
F^{-1}\{G(\kappa) ; \xi\}=g(\xi)=\frac{1}{2 \pi} \int_{-\infty}^{\infty} e^{i \kappa \xi} G(\kappa) d \kappa .
$$

The change of variables $e^{\xi}=x$ and $i \kappa=c-\eta$, where $c$ is a constant, in (11) and (12) lead to

$$
G(i \eta-i c)=\int_{0}^{\infty} x^{\eta-c-1} g(\ln x) d x
$$

and

$$
g(\ln x)=\frac{1}{2 \pi i} \int_{c-i \infty}^{c+i \infty} x^{c-\eta} G(i \eta-i c) d \eta .
$$

By putting $x^{-c} g(\ln x) \equiv f(x)$ and $G(i \eta-i c) \equiv f^{*}(\eta)$ in (13) and (14), we get the Mellin transform of $f(x)$ and the inverse Mellin transform as given by (6) and (7), respectively.

Fractional differential equations appear more and more frequently in different research areas and engineering applications. Recently, many important phenomena in the fluid mechanics, signal processing, control theory, systems identification, finance and fractional dynamics, physics and other areas have been described by fractional differential equations.

There are several definitions for derivative of arbitrary (fractional) order as those we customary refer to as Riemann-Liouville, Grünwald-Letnikov and Caputo fractional derivatives. 
The Riemann-Liouville fractional derivative of order $\alpha$ of a function $f(t)$ is defined as [16].

$$
{ }_{0} D_{x}^{\alpha} f(t)=\frac{d^{n}}{d x^{n}}\left[{ }_{0} D_{x}^{-(n-\alpha)} f(t)\right]
$$

where $n$ is positive integer, $n-1<\alpha \leq n$ and

$$
{ }_{0} D_{x}^{-(n-\alpha)} f(t)=\frac{1}{\Gamma(n-\alpha)} \int_{0}^{x}(x-\tau)^{n-\alpha-1} f(\tau) d \tau
$$

is the Riemann-Liouville fractional integral of $f(t)$ of order $n-\alpha$. The application of the Mellin integral transform (6) to (15) is given by the formula

$$
M\left\{t_{0}^{\alpha} D_{x}^{\alpha} f(t) ; \eta\right\}=\frac{\Gamma(1-\eta)}{\Gamma(1-\eta-\alpha)} f^{*}(\eta) .
$$

The Caputo fractional derivative of order $\alpha$ is defined as [3],

$$
D_{*}^{\alpha} f(t)= \begin{cases}\frac{1}{\Gamma(n-\alpha)} \int_{0}^{t} \frac{f^{(n)}(\tau)}{(t-\tau)^{\alpha+1-n}} & \text { if } n-1<\alpha<n \\ \frac{d^{n} f(t)}{d t^{n}} & \text { if } \alpha=n\end{cases}
$$

where $n>0$ is integer.

The method we follow makes the rule of the Laplace transform

$$
\bar{f}(s)=L[f(t) ; s]=\int_{0}^{\infty} e^{-s t} f(t) d t
$$

of the Caputo derivative (17) of key importance [16],

$$
L\left[D_{*}^{\alpha} f(t) ; s\right]=s^{\alpha} L[f(t) ; s]-\sum_{k=0}^{n-1} f^{(k)}(0) s^{\alpha-1-K},
$$

where $n-1<\alpha \leq n$. The one-parameter generalization of the exponential function was introduced by Mittag-Leffler [15] as

$$
E_{\alpha}(z)=\sum_{n=0}^{\infty} \frac{z^{n}}{\Gamma(\alpha n+1)}
$$

and further generalized by Agarwal [1], who introduced the two-parameter Mittag-Leffler function

$$
E_{\alpha, \beta}(z)=\sum_{n=0}^{\infty} \frac{z^{n}}{\Gamma(\alpha n+\beta)}, \quad \text { where } \alpha>0 \text { and } \beta>0
$$


The effect of the application of the Laplace transform (18) applied to the function (20) is given by the formula $[18,1.2 .2,(1.80)]$,

$$
L\left[t^{\alpha m+\beta-1} \frac{d^{m}}{d t^{m}} E_{\alpha, \beta}\left( \pm a t^{\alpha}\right) ; s\right]=\frac{m ! s^{\alpha-\beta}}{\left(s^{\alpha} \mp a\right)^{m+1}}, \quad \operatorname{Re} s>|a|^{\frac{1}{\alpha}} .
$$

\section{Mellin Transform Method for Solving Black-Scholes Equations}

In this section, we employ the Mellin transform for solving the Black-Scholes equation of both the European and the American put options.

Theorem 3.1. The boundary value problem for the Black-Scholes equation (1) for an European put option $p_{E}(S, t)$ with exercise price $K$, subject to boundary condition (2) has a unique solution of the form

$$
p_{E}(S, t)=\frac{S^{\left(\frac{1}{2}-\frac{r}{\sigma^{2}}\right)} e^{-\frac{1}{8}(T-t)\left(\sigma+\frac{2 r}{\sigma}\right)^{2}}}{\sigma \sqrt{2 \pi(T-t)}} \int_{0}^{K} \frac{(K-u) u^{\left(\frac{r}{\sigma^{2}}-\frac{3}{2}\right)}}{e^{\frac{1}{2}\left(\frac{\ln \frac{S}{u}}{\sigma \sqrt{T-t}}\right)}} d u .
$$

Proof. Denote the Mellin transform of $p_{E}(S, t)$ by $p_{E}^{*}(w, t)$. Application of the Mellin transform (6) to the equation (1) and the boundary conditions (2), leads because of (9) to the separable differential equation

$$
\frac{\mathrm{d} p_{E}^{*}(w, t)}{\mathrm{d} t}+\left[\frac{\sigma^{2}}{2} w(w+1)-r w-r\right] p_{E}^{*}(w, t)=0 .
$$

Its solution can be obtained readily and has the form

$$
p_{E}^{*}(w, t)=\theta^{*}(w) e^{\mu q(w)},
$$

where

$$
q(w)=w^{2}+\left(1-K_{1}\right) w-K_{1}, K_{1}=\frac{2 r}{\sigma^{2}}, \text { and } \mu=\frac{\sigma^{2}}{2}(T-t) .
$$

Since it is easy to see that

$$
M^{-1}\left\{\theta^{*}(w) ; S\right\}=\theta(S)=(K-S)^{+},
$$


we should focus on the determination of the inverse Mellin transform (7) of the function $e^{\mu q(w)}$. By the shifting property (8) and setting $\nu=\frac{1-K_{1}}{2}$, we get

$$
M^{-1}\left\{e^{\mu q(w)} ; S\right\}=e^{-\mu(\nu-1)^{2}} S^{\nu} f(S),
$$

where $f(S)=M^{-1}\left\{e^{\mu w^{2}} ; S\right\}$.

According to (12) and (14), by putting

$$
G(i w)=e^{\mu w^{2}}, \quad g(\ln S)=M^{-1}\{G(i w) ; S\},
$$

and substituting $e^{\xi}=S$ and $i \kappa=-w$, one can get [4, p. 611, Table B-1, (3)],

$$
g(\xi)=\frac{1}{2 \pi} \int_{-\infty}^{\infty} e^{i \kappa \xi} e^{-\mu \kappa^{2}} \mathrm{~d} \kappa=\frac{1}{2 \sqrt{\pi \mu}} e^{-\frac{\xi^{2}}{4 \mu}} .
$$

Hence,

$$
f(S)=g(\ln S)=\frac{1}{\sigma \sqrt{2 \pi(T-t)}} e^{-\frac{1}{2}\left(\frac{\ln S}{\sigma \sqrt{T-t}}\right)^{2}} .
$$

From (23), (24), (25), and (26), by the convolution theorem (10) it is possible to see indeed that the solution of the boundary problem stated is given by (22).

Theorem 3.2. The Black-Scholes equation (3) for the price of an American put option $p_{A}(S, t)$ with exercise price $K$ and critical stock price $S^{*}(t)$, subject to the time condition (4) and the smooth-pasting conditions (5) has a solution of the form

$$
p_{A}(S, t)=p_{E}(S, t)+r K \int_{t}^{T} M_{*}(S, t ; x) \mathrm{d} x,
$$

where

$$
M_{*}(S, t ; x)=\frac{e^{-\mu(\nu-1)^{2}\left[\frac{S(t)}{S^{*}(x)}\right]^{\nu}}}{\sigma \sqrt{2 \pi(x-t)}} \int_{0}^{1} e^{-\frac{1}{2}\left(\frac{\ln \left(\frac{1}{\xi} \frac{S(t)}{S^{*}(x)}\right)}{\sigma \sqrt{x-t}}\right)} \frac{\mathrm{d} \xi}{\xi^{1+\nu}}
$$

and

$$
K-S^{*}(t)=p_{E}\left\{S^{*}(t) ; t\right\}+r K \int_{1}^{T} M_{*}\left(S^{*}, t ; x\right) \mathrm{d} x
$$


Proof. Denote the Mellin transform of $p_{A}(S, t)$ and $f(S, t)$ by $p_{A}^{*}(w, t)$ and $f^{*}(w, t)$, respectively.

According to (9), the application of the Mellin transform (6) to the equation (3) results into the non-homogeneous linear differential equation

$$
\frac{\mathrm{d} p_{A}^{*}(w, t)}{\mathrm{d} t}+\left[\frac{\sigma^{2}}{2} w(w+1)-r w-r\right] p_{A}^{*}(w, t)=f^{*}(w, t),
$$

where

$$
f^{*}(w, t)=-\frac{r K}{w}\left[S^{*}(t)\right]^{w} .
$$

Since (29) is linear nonhomogeneous differential equation of the first order, we find straightforwardly its solution of the form

$$
p_{A}^{*}(w, t)=A(w) e^{-\frac{\sigma^{2}}{2} q(w) t}+\frac{r K}{w} \int_{t}^{T}\left[S^{*}(x)\right]^{w} e^{\frac{\sigma^{2}}{2} q(w)(x-t)} \mathrm{d} x,
$$

where as in Theorem 3.1, $q(w)=w^{2}+\left(1-K_{1}\right) w-K_{1}, K_{1}=\frac{2 r}{\sigma^{2}}$.

By setting $t=T$ both in (4) and (30), it can be seen that

$$
A(w)=\theta^{*}(w) e^{\frac{\sigma^{2}}{2} q(w) T},
$$

and hence, if $\mu=\frac{\sigma^{2}}{2}(T-t)$,

$$
p_{A}^{*}(w, t)=\theta^{*}(w) e^{\mu q(w)}+\frac{r K}{w} \int_{t}^{T}\left[S^{*}(x)\right]^{w} e^{\frac{\sigma^{2}}{2} q(w)(x-t)} \mathrm{d} x .
$$

By the inverse Mellin transform (7) and taking into account Theorem 3.1, the above equation reduces to (27), where

$$
M_{*}(S, t ; x)=\frac{1}{2 \pi i} \int_{\gamma-i \infty}^{\gamma+i \infty} \frac{1}{w} e^{\frac{\sigma^{2}}{2} q(w)(x-t)}\left[\frac{S(t)}{S^{*}(x)}\right]^{-w} \mathrm{~d} w,
$$

with $\gamma=\operatorname{Re} w$. The second term in (27) provides the early exercise premium. From (25) and (26) it follows immediately that if $\gamma=\frac{\sigma^{2}}{2}(x-t)$, then

$$
M^{-1}\left\{e^{\gamma q(w)} ; S\right\}=\frac{e^{-\mu(\nu-1)^{2}} S^{\nu}}{\sigma \sqrt{2 \pi(x-t)}} e^{-\frac{1}{2}\left(\frac{\ln S}{\sigma \sqrt{x-t}}\right)^{2}}
$$

where $\nu=\frac{1-K_{1}}{2}$. By using the well known fact that

$$
M^{-1}\left\{\frac{1}{w} ; S\right\}=\left\{\begin{array}{ll}
1, & 0 \leq S \leq 1 \\
0, & S>1
\end{array},\right.
$$


and the convolution theorem (10), we get

$$
M_{*}(S, t ; x)=\frac{e^{-\mu(\nu-1)^{2}\left[\frac{S(t)}{S^{*}(x)}\right]^{\nu}}}{\sigma \sqrt{2 \pi(x-t)}} \int_{0}^{1} e^{-\frac{1}{2}\left(\frac{\ln \left(\frac{1}{\xi} \frac{S(t)}{S^{*}(x)}\right)}{\sigma \sqrt{x-t}}\right)} \frac{\mathrm{d} \xi}{\xi^{1+\nu}} .
$$

Substituting now $S=S^{*}$ in (27) and taking into account (5), we obtain the integral equation (28) for the free boundary and the statement is proved.

\section{Computational Examples}

In this section, we compare our result (22) for the European put option prices with other results computed by different methods. Unfortunately, we were not successful in finding appropriate values of $S^{*}$ to test the result of Theorem 3.2 computationally. We leave it as an open problem to find examples that work out on the American put option.

Table 1 shows the results of our method compared to the analytical results of European put option values obtained in Table 1 of [6].

\begin{tabular}{|l|c|c|c|c||l|l|}
\hline \multicolumn{1}{|c|}{$r$} & $S$ & $K$ & $\sigma$ & $T$ & \multicolumn{1}{|c|}{$p$} & \multicolumn{1}{|c|}{$p_{E}$} \\
\hline 0.125 & 1 & 1 & 0.5 & 1 & 0.1327 & 0.132711 \\
0.08 & 1 & 1 & 0.4 & 1 & 0.117 & 0.11698 \\
0.045 & 1 & 1 & 0.3 & 1 & 0.0959 & 0.0959102 \\
0.02 & 1 & 1 & 0.2 & 1 & 0.0694 & 0.069359 \\
0.005 & 1 & 1 & 0.1 & 1 & 0.0373 & 0.0373341 \\
0.09 & 1 & 1 & 0.3 & 1 & 0.0761 & 0.0761239 \\
0.04 & 1 & 1 & 0.2 & 1 & 0.06 & 0.06004 \\
0.01 & 1 & 1 & 0.1 & 1 & 0.0349 & 0.0349022 \\
0.08 & 1 & 1 & 0.2 & 1 & 0.0442 & 0.0441747 \\
0.02 & 1 & 1 & 0.1 & 1 & 0.0304 & 0.0303685 \\
0.12 & 1 & 1 & 0.2 & 1 & 0.0317 & 0.0316846 \\
0.03 & 1 & 1 & 0.1 & 1 & 0.0263 & 0.0262643 \\
\hline
\end{tabular}

Table 1: A comparison of our method denoted by $p_{E}$ with the analytical results denoted by $p$.

In Table 2, we compare our method with three different techniques along with the true Black-Scholes price (in the second column) for an European put 
with fixed values of $K=50, r=0.05, \sigma=0.25$, and $T=3$. The inserted values in columns 3,4 , and 5 , are numerical values obtained from [18] as a benchmark to compare with our results.

\begin{tabular}{|c||l|l|l|l|l|}
\hline$S$ & BS & BT & MC & IE & Theorem 3.1 \\
\hline 10 & 33.0363 & 33.0362 & 33.0345 & 33.0369 & 33.0363 \\
15 & 28.0619 & 28.0618 & 28.0595 & 28.0629 & 28.0619 \\
20 & 23.2276 & 23.2273 & 23.2291 & 23.2300 & 23.2276 \\
25 & 18.7361 & 18.7362 & 18.7339 & 18.7390 & 18.7361 \\
30 & 14.7739 & 14.7736 & 14.7748 & 14.7749 & 14.7739 \\
35 & 11.4384 & 11.4388 & 11.4402 & 11.4402 & 11.4384 \\
40 & 8.7338 & 8.7352 & 8.7374 & 8.7348 & 8.73378 \\
45 & 6.6021 & 6.6025 & 6.6014 & 6.6012 & 6.60206 \\
50 & 4.9564 & 4.9556 & 4.9559 & 4.9563 & 4.95639 \\
55 & 3.7046 & 3.7073 & 3.7076 & 3.7042 & 3.7046 \\
60 & 2.7621 & 2.7640 & 2.7602 & 2.7612 & 2.76214 \\
65 & 2.0574 & 2.0592 & 2.0581 & 2.0571 & 2.05743 \\
70 & 1.5328 & 1.5346 & 1.5324 & 1.5325 & 1.53275 \\
75 & 1.1430 & 1.1427 & 1.1407 & 1.1426 & 1.14304 \\
80 & 0.8538 & 0.8549 & 0.8543 & 0.8537 & 0.85384 \\
85 & 0.6392 & 0.6401 & 0.6405 & 0.6391 & 0.639189 \\
90 & 0.4797 & 0.4803 & 0.4790 & 0.4794 & 0.47971 \\
\hline
\end{tabular}

Table 2: A comparison of the Black-Scholes (BS) of European put option prices including Bionomial Tree (BT), Monte-Carlo (MC), Implicit Euler (IE), and Theorem 3.1 with fixed values of $K=50, r=0.05$, $\sigma=0.25$, and $T=3$.

\section{Joint Transforms Method for Solving a Fractional Black-Scholes Equation}

For $0<\alpha \leq 1$ and $0<\beta \leq 1$, consider the fractional generalization of the equation (1) of the form,

$$
D_{*}^{\beta} p_{E}(S, t)+\frac{\sigma^{2}}{2} S^{\alpha+1}{ }_{0} D_{S}^{\alpha+1} p_{E}(S, t)+r S^{\alpha}{ }_{0} D_{S}^{\alpha} p_{E}(S, t)-r p_{E}(S, t)=0,
$$


in terms of the Caputo time-fractional derivative (17) and the Riemann-Liouville derivative (15). Evidently the equation (31) reduces to (1) when $\alpha=\beta=1$.

In this section, we provide a method based on joint application of the Mellin transform (6) and the Laplace transform (18), for deriving an integral form of the solution of the boundary value problem of (31) in terms of the oneparameter Mittag-Leffler function.

Theorem 5.1. For $0<\alpha \leq 1$ and $0<\beta \leq 1$, the boundary value problem for the fractional Black-Scholes equation (31) for a European put option $p_{E}(S, t)$ with exercise price $K$, subject to boundary conditions (2), has a solution of the form

$$
p_{E}(S, t)=\int_{0}^{K}(K-u) g\left(\frac{x}{u}\right) \frac{d u}{u},
$$

where $g(x)=M^{-1}\left\{\frac{E_{\beta}\left[q(w, \alpha) t^{\beta}\right]}{E_{\beta}\left[q(w, \alpha) T^{\beta}\right]} ; x\right\}$, and

$$
q(w, \alpha)=\frac{\Gamma(1-w)}{\Gamma(1-w-\alpha)}\left[\frac{\sigma^{2}}{2}(w+\alpha)+\frac{\Gamma(1-w-\alpha)-\Gamma(1-w)}{\Gamma(1-w)} r\right] .
$$

Proof. Application of the Mellin transform (6) to the equation (31) and the boundary conditions (2) leads because of (16) to

$$
D_{*}^{\beta} p_{E}^{*}(w, t)+\left[\frac{\sigma^{2}}{2} \frac{\Gamma(1-w)}{\Gamma(-w-\alpha)}+\frac{\Gamma(1-w)-\Gamma(1-w-\alpha)}{\Gamma(1-w-\alpha)} r\right] p_{E}^{*}(w, t)=0,
$$

where as in Theorem 3.1, $p_{E}{ }^{*}(w, t)$ is the Mellin transform of $p_{E}(S, t)$. By using the recurrence formula

$$
(-w-\alpha) \Gamma(-w-\alpha)=\Gamma(1-w-\alpha),
$$

we obtain the above equation in the form,

$$
D_{*}^{\beta} p_{E}^{*}(w, t)=q(w, \alpha) p_{E}^{*}(w, t),
$$

where

$$
q(w, \alpha)=\frac{\Gamma(1-w)}{\Gamma(1-w-\alpha)}\left[\frac{\sigma^{2}}{2}(w+\alpha)+\frac{\Gamma(1-w-\alpha)-\Gamma(1-w)}{\Gamma(1-w)} r\right] .
$$


The Laplace transform (18) applied to (33) implies according to (19),

$$
\bar{p}_{E}^{*}(w, s)=\bar{p}_{E}(w, 0) \frac{s^{\beta-1}}{s^{\beta}-q(w, \alpha)},
$$

where $\bar{p}_{E}^{*}(w, s)$ is the Laplace-Mellin transform of the European put option $p_{E}(S, t)$.

By the inverse Laplace transform applied to (34) and (21), we can see that

$$
p_{E}^{*}(w, t)=p_{E}(w, 0) E_{\beta}\left[q(w, \alpha) t^{\beta}\right],
$$

where $E_{\beta}$ is the one-parameter Mittag-Leffler function. From the boundary conditions it becomes clear that

$$
p_{E}^{*}(w, 0)=\frac{\theta^{*}(w)}{E_{\beta}\left[q(w, \alpha) T^{\beta}\right]},
$$

and hence

$$
p_{E}^{*}(w, t)=\theta^{*}(w) \frac{E_{\beta}\left[q(w, \alpha) t^{\beta}\right]}{E_{\beta}\left[q(w, \alpha) T^{\beta}\right]} .
$$

The latest equation means that

$$
p_{E}(S, t)=\frac{1}{2 \pi i} \int_{\gamma-i \infty}^{\gamma+i \infty} \theta^{*}(w) \frac{E_{\beta}\left[q(w, \alpha) t^{\beta}\right]}{E_{\beta}\left[q(w, \alpha) T^{\beta}\right]} S^{-w} d w,
$$

where $\gamma=\operatorname{Re} w$. According to the convolution theorem (10), the equation (36) leads to the representation (32), where

$$
g(x)=M^{-1}\left\{\frac{E_{\beta}\left[q(w, \alpha) t^{\beta}\right]}{E_{\beta}\left[q(w, \alpha) T^{\beta}\right]} ; x\right\},
$$

and the proof is accomplished.

Remark 5.2. In the case $\alpha=\beta=1$, the representation (32) reduces to (22).

\section{Acknowledgments}

We are pleased to express our appreciation to our teacher, colleague and friend Prof. Dr. Shyam L. Kalla, Department of Computer Engineering - VIHE Jodhpur, India, for his vital ideas and constant support to our research. 


\section{References}

[1] R. P. Agarwal, A propos dune Note M. Pierre Humbert, C. R. Acad. Sci. Paris 236 (1953), 2031 - 2032.

[2] F. Black and M. Scholes, The Pricing of Options and Corporate Liabilities, Journal of Political Economy 81, 3 (1973), 637 - 654.

[3] M. Caputo, Linear Models of Dissipation whose Q is almost Frequency Independent-II, Geophysical J. International of the Royal Astronomical Soc. 13 (1967), 529 - 539.

[4] Z. Ali Elshegmani and R. Rozita Ahmed, Analytical Solution for an Arithmetic Asian Option Using Mellin Transforms, Int. Journal of Math. Analysis 5 (2011), 1259 - 1265.

[5] A. Erdelyi, W. Magnus, F. Oberhettinger, and F. G. Tricomi, Tables of Integral Transforms. McGraw-Hill, New York, USA, (1954).

[6] R. Geske and H. E. Johnson, The American Put Option Valued Analytically, The Journal of Finance 39, 5 (1984), 1511 - 1524.

[7] P. Glasserman, Monte Carlo Methods in Financial Engineering, Applications of Mathematics, Stoch. Model. Appl. Probab., Springer-Verlag, New York (2004).

[8] L. Jodar, P. Sevilla-Peris, J. C. Cortes, and R. Sala, A new direct method for solving the BlackScholes equation, Applied Mathematics Letters 18, 1 (2005), $29-32$.

[9] I. Kim and S. Byun, Optimal exercise boundary in a binomial option pricing model, Journal of Financial Engineering 3 (1994), 137 - 158.

[10] S. Kumar, A. Yildirim, Y. Khan, H. Jafari, K. Sayevand, and L. Wei, Analytical solution of fractional Black-Scholes equation European option pricing equation by using Laplace transform, Journal of Fractional Calculus and Applications 2, 8 (2012), 1 - 9.

[11] M. Lauko and D. Sevcovic, Comparison of numerical and analytical approximations of the early exercise boundary of American put options, ANZIAM Journal 51 (2010), 430 - 448. 
[12] F. A. Longstaff and E. S. Schwartz, Valuing American options by simulation: a simple least-squares approach, Review of Financial Studies $\mathbf{1 4}$ (2001), 113 - 147.

[13] O. I. Marichev, Handbook of Integral Transforms of Higher Transcendental Functions: Theory and Algorithmic Tables, Ellis Horwood Ltd., Chichester (1982).

[14] R. C. Merton, Theory of rational option pricing, Bell J. Econ. Manag. Sci. 4, 1 (1973), 141183.

[15] G. M. Mittag-Leffler, Sur la nouvelle fonction $E_{\alpha}(x)$, C. R. Acad. Sci. Paris 137 (1903), 554 - 558.

[16] I. Podlubny, Fractional Differential Equations, Academic Press, San Diego, CA (1996).

[17] A. P. Prudnikov, Y. A. Brychkov, and O. I. Marichev, Integrals and Series. Volume 3: More special functions, Gordon and Breach Science Publishers (1990).

[18] Mark Richardson, Numerical Methods for Option Pricing, University of Oxford, Special topic (2009).

[19] L. Rogers, Monte Carlo valuation of American options, Math. Finance 12, 3 (2002), $271-286$.

[20] J. Tsitsiklis and B. Van Roy, Regression methods for pricing complex American-style options, IEEE Transactions on Neural Networks 12 (2001), 694703.

[21] T. A. Vasileva and O. E. Vasileva, Mellin transform to the Black-Scholes equations, Journal of VolGU \#1: Mathematics, Physics 12 (2009), 55 63. 
